\title{
燕窝糖肽的分离与鉴定
}

\author{
童 坦 君 \\ 刘德 勇 \\ （北京医学院生物化学教研空）（美国生物制品标准局生物化学与生物物理研究部）
}

一百年前德国生物化学界的先驱 Hoppe-Seyler 氏曾对食用燕窝（Edible bird's nest）进行 了研究, 从其物理性能与还原性两方面认识到它的本质是粘蛋白 ${ }^{[1]}$. 燕窝由东南亚一带常见 的雨燕 (Collocalia 属)的唾液腺分泌产物所形成. 所以由燕窝制备而成的糖蛋白也称为雨燕类 粘蛋白 ${ }^{[2-4]}$. 与牛、羊领下腺的粘蛋白等其它粘蛋白相比, 目前对燕窝粘蛋白的结构所知甚少, 这主要是分离、提纯其组成成份十分困难所致。

本文用交联葡聚糖 G50 超细颗粒长柱（柱高 192 厘米）结合高分辩率的蛋白质高效液相 色谱 $\mathrm{I}_{60}$ 柱, 从燕窝的胃蛋白酶消化物(简称燕窝制品)中成功地分离到一种糖肽, 并对此进行 了篮定.

\section{实 验材料与方法}

实验用燕窝胃蛋白酶消化制品根据文献 [?]制备. 胃蛋白酶消化的燕窝为市售.

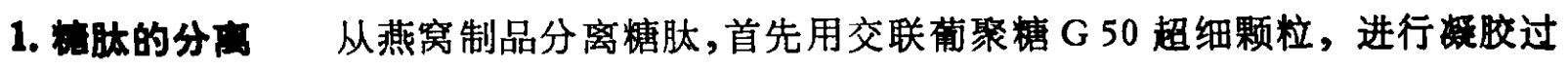
滤，其条件详见图 1 及其附注. 按图 1 中峰段收集组分 A、B、C、D. 然后将组分 A 冷冻干燥 后, 以溴化気裂解. 裂解后用 Waters Associates 204 型高效液相色谱仪, 以蛋白质高效液相色 谱层析柱 $\mathrm{I}_{\rho}$ 再加以分离. 其层析条件详见图 3 及其附注.

2. 分析方法 1) 氨基酸与氨基糖的分析按前法 ${ }^{[5]}$. 用 Beckman $121 \mathrm{M}$ 型氨基酸分析仪 分析之.

2) 中性糖的分析：将含糖 1-10 微克的样品溶于 480 微升去离子水中, 加 $4 N$ 甲曝酸 20 微升, Dowex $50 \times 8\left(200-400\right.$ 目, $\mathrm{H}^{+}$型) 树脂 10 毫克, 充以氮气后, $100{ }^{\circ} \mathrm{C}$ 水解 20 小时. 水 解后, 水解液用 $4 \mathrm{~N} \mathrm{NaOH}$ 中和, 再加以 20 微升 $3 \mathrm{~N} \mathrm{NaOH}$ 后, 用 Millipore 滤器 (0.45 微米) 滤 过, 然后在真空离心机 (Savant, SVC-100 型)中离心抽干. 干燥后, 加 3 至 4 体积的 $90 \%$ 乙醇 更新溶解之. 以上径处理的水解液按前法 ${ }^{[6]}$ 在 $\mathrm{Li}^{+}$型氨基酸分析柱上以反向层析法分析之, 用 $90 \%$ 乙醇为洗脱液. 另一份等体积经处理的水解液, 则以葡萄糖化酶处理之 ${ }^{[6]}$, 然后上柱以 监别惐萄糖与甘露糖.

3）唾液酸分析法: 样品液 $(0.1 \mathrm{ml})$ 置于试管中冻干之. 干燥后加 $2 N$ 甲碏酸甲酻溶液 $0.5 \mathrm{ml}$, 然后充以气，将试管加盖密封后 $65^{\circ} \mathrm{C}$ 保温 24 小时. 然后于 $40^{\circ} \mathrm{C}$ 用将气其吹干. 吹干后加 $1.0 \mathrm{~N} \mathrm{NaOH} 1.10$ 毫升, $2^{\circ} \mathrm{C}$ 放置 60 分钟, 用两倍体积的水稀释之. 用 Beckman $121 \mathrm{M}$ 型虫基酸分析仪测定唾液酸含量 ${ }^{[7]}$. 以 $\mathrm{N}$-酰神经氨酸作为唾液酸的标准, 与样品同样 处理.

4) 聚丙烯酰胺凝胶电泳: 圆盘电泳系按 Fairbanks 等氏法 ${ }^{[0]}$ 在十二烷基硫酸钠(SDS) 存 在条件下进行. 嘹胶浓度为 $7.5 \%, \mathrm{pH}$ 为 7.4。糖肽的 SDS 聚丙烯酰胺凝胶平板电泳与硝酸 银染色法,按文献 [11]进行.

本文 1984 年 1 月 25 日收到. 
3. 化学处玨 1)溴化氧裂解法: 按文献 [8]方法裂解；2)糖肽的去唾液酸：按 Farrar 氏法 ${ }^{[9]}$ 进行.

\section{结果与讨论}

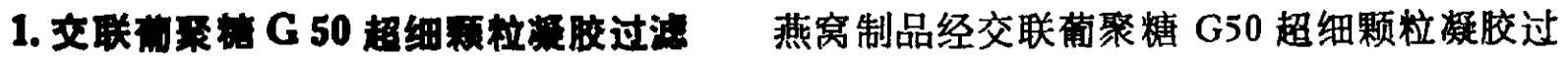

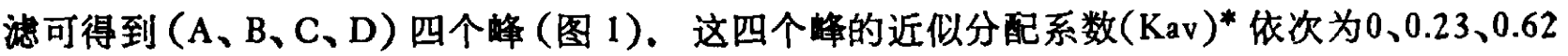
与 0.82 . 组分 $\mathrm{A}$ 为燕窝制品中分子量较大的部分，按重量计占洗脱下来的物质总量的 $58.1 \%$.

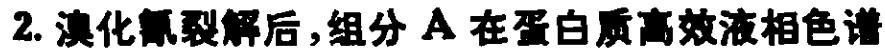

\section{L) 柱上的层析行为 组分 $\mathrm{A}$ 是燕宽制品经 交联葡} 聚糖 G50凝胶过滤后的主要组分.

与其起始物质燕窝制品相似，组分 A 如不去除其 唾液酸部分，电泳时常浓集于电泳前沿。此时电泳的 分辨率几乎等于零。在去除唾液酸部分后，燕窝制品 与组分 A 的 SDS 聚丙烯酰胺凝胶电泳的分辩率大为 提高. 用胶浓度为 $7.5 \%$ 的 Fairbanks 氏法 ${ }^{[10]}$ 进行电 泳，可见燕窝制品呈数条区带 (图 2)，但组分 A 仅呈 二条带, 其一较为浓集. 如前所述组分 $A$ 在交联葡聚 糖 $\mathrm{G} 50$ 柱中 恰好出现在一个外水体积 $\left(\mathrm{V}_{0}\right)$ 处. 如 将组分 $\mathrm{A}$ 的溶液用蛋白质高效液相层析 $\mathrm{I}_{60}$ 柱进行分 离, 流洗速度为每分钟 1 裹升, 则在样品注人后, 仅在

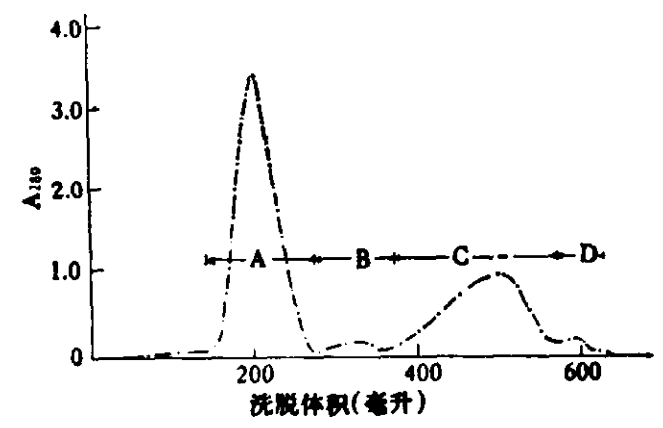

图 1 燕窝制品的交联薄聚糖 G50 挍胶法过

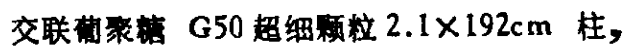
用 $0.1 \mathrm{M} \mathrm{pH} 5.0$ 醋酸铵溶液事先平街并洗脱 之. 样品 500 毫克溶于 5-10 毫升该缓冲液 后上样, 流洗速度为 5.5 竞开/小时 第 6 分钟出现一单一的峰 (图 3a). 此峰亦即出现于相当一个外水体积 $\left(V_{0}\right)$ 处.

在用溴化気裂解后,组分 A 用同样条件进行层析, 可见六个峰 (图 3b). 在一个外水体积处 (注人后第6分钟)，仍有一个峰 $\left(A_{1}\right)$ ，但此峰比等浓度未经处理的组分 $A$ 要小得多，可能是未 裂解的组分 $\mathrm{A}$, 或为裂解后分子量仍然较大的碎片. 第二个峰 $\left(\mathrm{A}_{2}\right)$ 紧接在该峰之后, 出现于 样品注人的第 7 分钟. 此是裂解后的组分 $\mathrm{A}$ 的主要吸收峰之一. 色谱中最大的吸收峰是第五 蜂, 然而经检验后发现其稭留时间与氨基酸(如苯丙氨酸) 完全相同, 出现于注入后的第 16 分 钟. 可见其已是小分子物质, 而非裂解后的主要糖肽片段, 在澳化袅裂解后, 如即以去离子 水透析样品, 然后冷冻干燥, 以同法用 $I_{6}$ 柱进行层析, 仅出现 $A_{1}$ 与 $A_{2}$ 两个峰. 可见 $A_{2}$ 是组 分 $\mathrm{A}$ 经滨化氞裂解后,较为主要的糖肽片段。

我们用伴刀豆球蛋白一琼脂糖 (ConA-Sepharose 4B) 亲和层析柱分离组分 A 的溴化氨裂解 物, 未获成功,乃将上述分析时所用高效液相色谱法应用于分离, 终于成功分离得到糖肽 $A_{2}$.

3. 机肽 $A_{2}$ 的生化紧性质 以上高效液相色谱分段分离方法经加大样品注入量,并反 复进行之. 收集 $A_{2}$ 峰段,冷冻干燥。将该 $A_{2}$ 样品(糖朋 $A_{2}$ ) 用同法进行层析, 可见呈单一的洗 眖峰(图4). 对照图 $2 b$ ，可见该峰出现的位置，即是其原第二峰 $\left(A_{2}\right)$ 的位置.

利用平板 SDS 聚丙烯酰胺胶电泳及比考马斯亮蓝法更为灵敏的硝酸银染色法 ${ }^{[11]}$, 去唾 液酸后的燕窝制品呈八条以上的区带,但榶肽 $\mathrm{A}_{2}$ 仅呈其迁移速度比燕窝制品大多数区带快的 一条浓集区带(图 5) 从以上电泳图像(图 5) 与蛋白质高效液相色谱 $\mathrm{I}_{60}$ 柱层析图像（图 4)看，

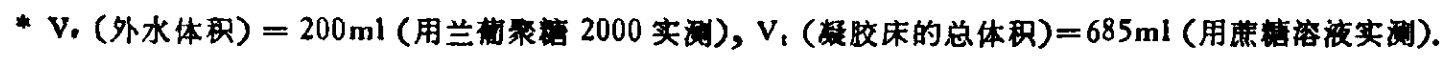


图 2 去唾液酸后的燕宽制品(a)与其组分 A(b) 的 SDS 聚丙烯酰胺凝胶电泳 ${ }^{[10]}$ 图像

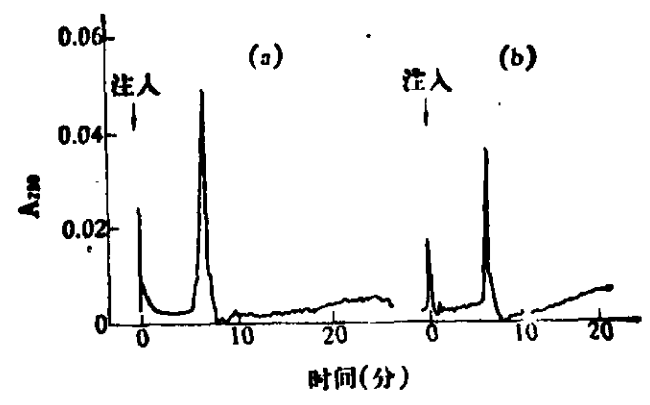

图 4 糖肽 $A_{2}$ 在蛋白质高压液相色谱 $I_{60}$ 柱上的层析行为

样品: 30 微克/ $/ \mathrm{ml}$,(a) 20 微升，(b) 15 微开. 洗脱缓冲液: $0.01 \% \mathrm{NH}_{4} \mathrm{HCO}_{3}, \mathrm{pH} 7.0$. 流速: 1.0 管升/ 分.

$\mathrm{A}_{240}$ ：洗脱液在 $280 \mathrm{~nm}$ 的光吸收

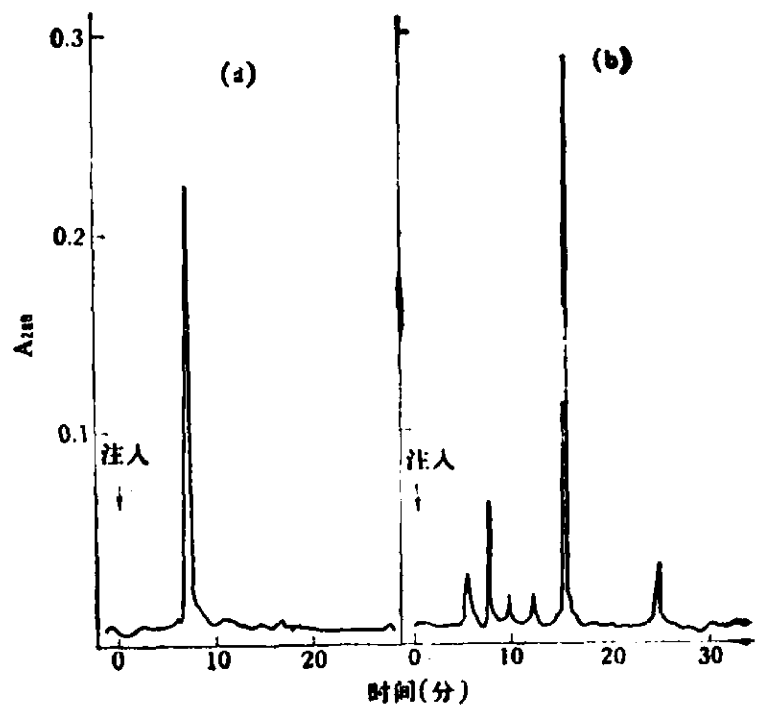

图 3 䜩宽制品的组分 A 澳化風裂解前(2)后 (b)在蛋白质高效液相色谱 $\mathrm{I}_{60}$ 柱上的层 析行为的变化

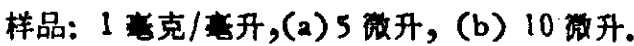
缕冲液: $0.01 \% \mathrm{NH}_{4} \mathrm{HCO}_{3}, \mathrm{pH} 7.0$. 流速: 1.0 莫开/分

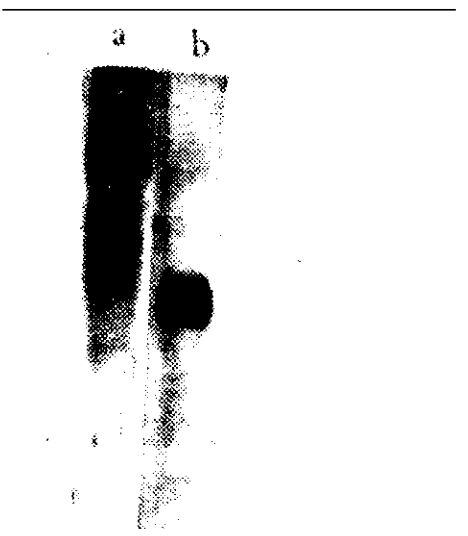

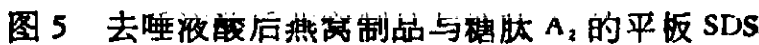
聚丙烯酰胺凝胶电泳(硝䣨银染色法)图隐

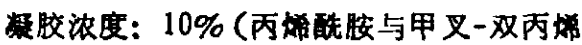
酰胺的比例为 $37.5: 1$ ).

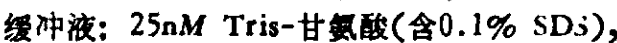
pH 8.3

可以认为用上法分离所得的糖肽 $A_{2}$ 已近于单一成分.

进一步实验发现糖肽 $\mathrm{A}_{2}$ 的 SDS 聚丙烯酰胺凝胶电泳速度接近，但稍高于大豆肤蛋白酶抑 制物(分子量 21,000), 且其在具有分子篇性能的 $I_{60}$ 柱(最大分离范围为分子量 20,000) 上的殓 留时间(7 分钟)，紧接于 $V_{0}$ (6 分钟)之局(图 3、图 4). 所以佔计其分子量接近于 20.000 。但 
准确测定其分子量尚有一定困难,因含糖较多的蛋白质, 其 SDS-聚丙烯酰胺㢸胶电泳的行为 与一般蛋白质不同,泳动速度常有异于同分子量的单纯蛋白质 ${ }^{[12]}$. 氨基酸分析显示,糖肽 $\mathrm{A}_{2}$ 所 含酸性氨基酸多于碱性氨基酸. 其所含丝、甘、谷、天门冬等氨基酸皆甚为丰富. 与丝氨酸同 样, 在多种粘蛋白中常籍氧一糖苷键与糖相连的另一种氨基酸, 苏氨酸的含量也不低.

表 1 精肽 $A_{2}$ 及燕窝制品中氨基酸的组成 $(\%) *$

\begin{tabular}{|c|c|c|c|c|c|}
\hline 虫基酸残基 & 燕窝制品 & 䤉朋 $\mathrm{A}_{2}$ & 基酸残基 & 燕宽制品 & 謤肽 $\mathrm{A}_{2}$ \\
\hline 酪 & 5.6 & 4.4 & 脯 & 9.5 & 7.0 \\
\hline 苯丙 & 6.1 & 3.7 & 甘 & 7.2 & 12.3 \\
\hline 赖 & 3.6 & 2.9 & 丙 & 5.1 & 7.8 \\
\hline 组 & 2.8 & 4.4 & $1 / 2$ 䁳 & 0.8 & 0.1 \\
\hline 精 & 5.9 & 3.6 & 纯 & 7.3 & 5.9 \\
\hline 天/天肢 & 9.9 & 8.2 & 蛋 & 0.5 & 0.5 \\
\hline 苏 & 6.5 & 6.9 & 异亮 & 3.1 & 2.6 \\
\hline 丝 & 10.5 & 12.8 & 亮 & 8.1 & 6.6 \\
\hline 谷/谷胺 & 7.3 & 10.1 & 色 & - & - \\
\hline
\end{tabular}

* 表中数字表示每百个氮基酸残基中该全基酸残基所占比例.

在糖肽 $\mathrm{A}_{2}$ 中还含有重量百分率为 $5.25 \%$ 的甘客糖或葡萄糖, 未发现其它中性糖的明显存 在,此点与含半乳糖很多的燕窝制品不同 (表 2). 与燕窝制品相似,糖肽 $\mathrm{A}_{2}$ 中氨基已糖的含量 相当丰富,氨基薄萄糖与氨基半釈糖的重量百分含量依次为 $6.0 \%$ 与 $6.2 \%$.

表 2 糖肽 $A_{2}$ 及燕窝制品中的糖含量 ${ }^{*}$

\begin{tabular}{|c|c|c|c|c|c|}
\hline & 燕宽制品 & 楛肽 $\mathrm{A}_{2}$ & & 燕密制品 & 榶肽 $\mathrm{A}_{2}$ \\
\hline 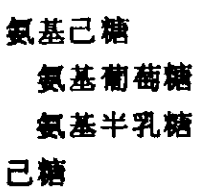 & $\begin{array}{l}3.3 \\
3.0\end{array}$ & $\begin{array}{l}6.0 \\
6.2\end{array}$ & 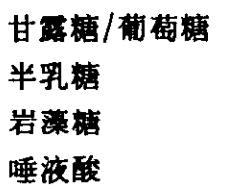 & $\left|\begin{array}{c}0.8(甘) ;<0 . Y(\text { 㳦 } \\
10.2 \\
0.4 \\
0.5\end{array}\right|$ & $\begin{array}{c}5.3 \text { (甘或蒲) } \\
<0.1 \\
<0.1 \\
\text { 末 测 }\end{array}$ \\
\hline
\end{tabular}

*表中数字表示每百克样品中的克数.

由于本文所用材料为燕窝酶解制品,杂有极少量的胃蛋白酶. 糖肽 $\mathrm{A}_{2}$ 有否可能即是胃蛋 白酶?这种可能性不大. 由于胃蛋白酶分子量 (33,000) 大于 $I_{60}$ 柱的分离范围 (分子量 $1,000-$ $20,000)$ ，而 $\mathrm{A}_{2}$ 峰的䄸留时间超过了一个外水体积. 再者,胃蛋白酶为单纯蛋白质, 而糖肽 $A$ 含糖量如此高,所以难以设想本文分离而得的糖肽 $A_{2}$ 来自胃蛋白酶,而非来自燕窝.

燕窝糖肽的分离成功,将为开展牛、羊领下腺来源以外的某些粘蛋白的结构研究,特别是 粘蛋白中糖与肽的联接方式的研究, 提供进一步的可能.

\section{考文献}

[1] Gottschalk, A. ed., in Glycoprotein, Elsevier Publishing Company, Amsterdam, 1972, Part A, 1-23.

[ 2$]$ Howe, C. et al., Arch. Biochem. Biophys., 95(1961), 512.

[ 3 ] Kathan, R. H. and Weeks, D. I., Arch. Biochem. Biophys., 134(1969), 572.

[ 4 ] Biddle, F. and Belyavin, G., J. Gen. Microbiol., 31(1963), 31.

[ 5 ] Liu, T. Y. and Chang, Y. H., J. Biol. Chem., 246(1971), 2842.

[ 6 ] Boykins, R. A. and Liu, T. Y., J. Biochem. Biophys. Methods, 2(1980), 71.

[ 7 ] Liu, T. Y., Methods Enzymol., 28(1972), 48.

[ 8 ] Gross, E. and VYitkop, B., J. Biol. Chem., 237(1962), 1856.

[9] Farrar, G. H. et al., Hoppe-Seyler's Physiol. Chem., 361(1980), 473.

[10] Fairbanks, G. et al., Biochem., 10(1971), 2606.

[11] Switzer, K. C. et al., Anal. Biochem., 98(1979), 231.

[12] Sharon, N., Complex Carbohydrates, Addison-Wesley Publishing Co., Massachsetts, 1978, 51-52. 